\title{
Arbor
}

\section{Las ciencias físico-químicas y su enseñanza}

José Aguilar Peris

Arbor CLXXIII, 681 (Septiembre 2002), 153-164 pp.

El principal objetivo de la educación es enseñar a los alumnos los métodos para la realización de su actividad fundamental: el estudio. Es evidente que toda persona que desea ejercer una actividad profesional -oculista, músico o electricista-debe desarrollar las técnicas de aprendizaje apropiadas bajo una dirección y unas normas rigurosas y didácticas. Un requisito primario para cumplir este objetivo es poseer una actitud mental afectiva hacia la profesión en cuestión. El mismo requisito debe cumplir el profesor hacia la materia que debe impartir. Indudablemente los firmes propósitos no bastan, si no van acompañados de un trabajo coordinado, constante y firme entre profesor y alumno (Chapman, 1959). El propósito de este artículo es adecuar estas ideas a las peculiaridades de las ciencias físico-químicas y su enseñanza, de cara al nuevo siglo (y nuevo milenio), recapitulando sobre las experiencias del pasado e intentando superarlas con nuevas técnicas pedagógicas que reflejen el nuevo espíritu del tiempo en que vivimos.

\section{Un recuerdo del PSSC}

Hace ya muchos años publicamos en esta misma Revista (Aguilar, 1965) un artículo sobre la enseñanza de la física que tenía como objetivo ofrecer nuestra opinión sobre un texto estadounidense, Curso de Física del PSSC (Physical Science Study Committee), que pretendía ser un 
ejemplo actualizado de la didáctica de esta disciplina, en el que habían intervenido centenares de personas, en su mayor parte, profesores de física de enseñanza media y universitaria, durante un periodo superior a cuatro años. En la década 1960-70 fue un libro revolucionario que introdujo un espíritu nuevo en la enseñanza de la física. Las viejas agrupaciones estancas de la física -mecánica, calor, acústica, óptica y electricidad- con un apéndice de física moderna, desaparecían para ser sustituidas, no como un conglomerado de fenómenos, sino como un proceso continuo, subrayándose la universalidad de sus leyes y la unidad de la física en todos sus aspectos. Los temas tratados estaban ordenados y relacionados, desde los más simples a los más complejos, incluyendo datos sobre el desarrollo histórico de las ideas básicas, la lucha en el pasado y los pasos que tuvieron que dar algunos cientí.icos para enunciar los principios fundamentales de la física.

El texto se complementaba con un libro de prácticas de laboratorio y un equipo de aparatos modernos y económicos, un gran número de películas científicas, una serie de publicaciones escritas por especialistas sobre temas de divulgación y una guía para el profesor. Existían dos niveles del texto, uno destinado a la enseñanza media y otro a la física general universitaria. El curso PSSC costó de 8 a 10 millones de dólares (de la época). El proyecto era acertado y tuvo una gran difusión, tanto en España como en Sudamérica. Se tradujo a múltiples idiomas (español, italiano, francés, portugués, japonés, sueco, etc.). Sólo tenía un defecto. $\mathrm{Al}$ menos en España, no se ajustaba a los programas académicos de física de los años $60 \mathrm{y}$, como muchos de los proyectos ambiciosos de este estilo, era un libro excelente para completar la formación de los profesores de física media y universitaria, pero que éstos no podían impartir a sus alumnos, especialmente porque carecían en sus horarios oficiales del tiempo necesario para su desarrollo. Las películas didácticas y los libros de prácticas y demás complementos se ensayaron en algunos centros pilotos que se habilitaron con estos fines. Se hicieron varias ediciones sucesivas del PSSC y finalmente desapareció de los catálogos editoriales y fue sustituido por otros proyectos de la misma índole.

\section{2. ¿Por qué estudiamos las ciencias físico-químicas?}

La física es la ciencia básica de la naturaleza. Estudia sus leyes universales e intenta describir y entender las propiedades y el comportamiento de la materia y la energía. La química es la rama de la ciencia que trata de la constitución, propiedades y transformaciones de la materia, 
así como de su estructura atómico-molecular. La relación entre ambas ciencias es tan íntima que existen disciplinas específicas como química física, física de los materiales, física del estado sólido, electroquímica, bioquímica, biofísica, termoquímica, etc, que son auténticas simbiosis.

Las aplicaciones de la física y la química en la medicina y la farmacia son innumerables. Existe un libro clásico, titulado Medical Physics (Glasser, 1944) de 1744 páginas en donde se discuten unas 300 aplicaciones físico-químicas en temas relacionados con las ciencias de la salud, tales como mecanismos y defectos de la visión y la audición, metabolismo químico de los alimentos, presión arterial, viscosidad de la sangre, presión osmótica en las células, capilaridad de los vasos sanguíneos, tensión superficial y función respiratoria, la bomba sodio-potasio, los ultrasonidos, las propiedades eléctricas de las fibras nerviosas, los rayos $\mathrm{X}$, el uso de trazadores radiactivos, etc. Comentando este libro, S. Chapman decía: «Durante varios años ofrecí pagar medio dólar a cualquiera de mis alumnos que fuese capaz de encontrar 10 páginas consecutivas de un texto general de física universitaria, entre las cuales no encontrasen nada aplicable a la profesión médica» (Chapman, 1959). En textos más modernos de física médica han ido incluyéndose nuevas aplicaciones a la medicina y a la biología, (Bouyssy, 1987) como la resonancia magnética nuclear, tomografías, microfotografías de neuronas, cámaras de positrones, contadores de centelleo, fotomultiplicadores para exploraciones cerebrales, difusión y difracción de neutrones, etc. Es cierto que las aplicaciones fisico-químicas a la ingeniería y a la telecomunicación son más directas que las correspondientes a las ciencias de la salud, pero no menos importantes.

\section{La enseñanza experimental de las ciencias físico-químicas}

Aunque la física es una disciplina del saber humano que se enseñaba ya en tiempos de Aristóteles (siglo IV a.C.) y en las primeras universidades que surgieron al final del Medievo, la verdad es que en sus comienzos no fue más que «filosofía natural», y hubo que esperar la aparición de Galileo y la introducción del método experimental para que se reconociera su valor como una ciencia de la naturaleza, en donde la observación, la medida y el razonamiento matemático van sustancialmente unidos. El estudio de los fenómenos naturales exige un diálogo vivo entre los físicos y la naturaleza, diálogo que se reduce casi siempre a observar, medir, razonar, calcular, comprobar y volver a observar, medir... El conjunto de estas etapas es la experimentación. Pero este método no se lleva a la Uni- 


\section{José Aguilar Peris}

versidad hasta el siglo XIX, cuando comienzan a surgir los primeros laboratorios de física experimental y se sentaron las bases de las investigación moderna. La Universidad de Cambridge nos dio el modelo con su célebre Cavendish Laboratory, en donde se practicaba simultáneamente la enseñanza y la investigación. Su primer director fue Maxwell y desde entonces fue y sigue siendo unos de los centros más fructíferos de la física mundial, puesto de manifiesto por el gran número de Premios Nobel otorgados a sus investigadores durante el siglo XX.

La química, como ciencia experimental (prescindimos de la antigua alquimia, esotérica, cuyo objetivo casi único era la transmutación de la materia en oro, la piedra filosofal, el elixir de la juventud, etc.) surgió en el siglo XVIII con los trabajos de Black, Cavendish, Priestley y especialmente del químico físico Antoine Laurent Lavoisier, padre de la química moderna y quien desde el comienzo de sus investigaciones reconoció la importancia de las mediciones precisas que realizaba siempre con gran exactitud. Estableció la naturaleza de la combustión, la composición del aire y del agua y estableció el principio universal de conservación de la masa, piedra angular de la química del siglo XIX. Los químicos aceptaron las ideas y métodos de Lavoisier, que coincidían en líneas generales con las planteadas por Galileo en el campo de la física.

Hoy el profesor de cualquier disciplina científica o tecnológica conoce bien el valor didáctico de los medios audiovisuales y lo utiliza a través de diapositivas, transparencias, películas, vídeos, ordenadores, etc. Sólo una experiencia magistral de cátedra o el trabajo serio del alumno en el laboratorio puede superar al medio audiovisual. El viejo aforismo: «escucha y olvidarás, observa y recordarás, realiza y entenderás», sigue siendo de actualidad en la enseñanza de las ciencias físico-químicas o tecnológicas.

Las demostraciones y experiencias de cátedra y laboratorio constituyen la mejor ayuda del profesor para que el alumno desarrolle su mente hacia la comprensión de un concepto físico y su relación con el mundo real. A pesar de ello, existen muchos argumentos en contra de estas demostraciones, tales como: «no tenemos presupuesto para ello», «se requiere mucho tiempo para su preparación», «hace falta personal auxiliar bien preparado», etc. Cierto es que algunas de estas afirmaciones son reales, pero los resultados que se obtienen merecen la inversión del tiempo utilizado, y muchas demostraciones de leyes o propiedades de la materia pueden realizarse con aparatos muy simples y de bajo precio. Con ello combatimos además el síndrome de que « la física y la química son muy difíciles», tan extendido entre los estudiantes. Cuando un concepto puede demostrarse mediante el uso de materias simples, no puede ser 
difícil de entender. Por ejemplo, el estudio termodinámico de la tracción de un hilo puede comprobarse experimentalmente tomando un pequeño aro de caucho en contacto con los labios (que son muy sensibles a los cambios de temperatura) y estirándolo rápidamente con las dos manos se detecta sensiblemente un calentamiento del caucho. Si bruscamente volvemos a la longitud inicial, se detecta un enfriamiento. Con estos antecedentes, la interpretación entrópica del proceso es inmediata y tiene todas las posibilidades de ser comprendida hasta por los alumnos más distraídos.

Hay que hacer constar que al hacer estas manifestaciones no queremos restringirnos a las ciencias físico-químicas que se imparten a nivel de enseñanza media o primer curso de las facultades universitarias, donde realmente la experimentación debe jugar un papel preponderante. Las técnicas experimentales deben desarrollarse igualmente en los cursos avanzados. Hay excelentes experiencias catalogadas en el campo de la física superior que utilizan la holografía, los rayos $\mathrm{X}$, el cuanto de acción de Planck, la medida de la velocidad de la luz o la medida de la carga eléctrica elemental. Igualmente, en el campo de la química avanzada pueden realizarse experiencias de laboratorio con métodos cromatográficos y espectroscópicos, electroquímica, radiactividad, síntesis de compuestos orgánicos, microscopía electrónica de barrido, metalurgia, nuevos materiales, etc.

Los objetivos fundamentales de estos trabajos experimentales son:

1. Introducir en el alumno el significado del enfoque experimental mediante el contacto y la ejecución de las manipulaciones necesarias. Es obvio que el estudiante cree en la estadística gaussiana más fácilmente cuando él acumula datos que cuando se le dice que aquellas medidas siguen una distribución gaussiana.

2. Introducir al alumno en los métodos de análisis de datos, tan frecuentes en el mundo de la ciencia y la ingeniería, y desarrollar la «conciencia del error», de modo que el futuro científico sea conocedor del valor relativo de sus medidas.

3. Apreciar el uso válido de las aproximaciones y no considerarlas como un método cómodo de obtener una respuesta fácil de un problema difícil ante la falta de una técnica apropiada. Este punto está en el corazón de nuestra filosofía sobre la enseñanza del análisis de datos. Consideramos que el fin no justifica los medios en ningún caso, $\mathrm{y}$, por tanto, tampoco en las ciencias experimentales. Además existe el peligro de que la aplicación de métodos incorrectos para hacer más «tratable» un problema difícil dé lugar a una solución completamente falsa. 
4. Conceder la máxima importancia a los métodos de registro gráfico, análisis por diferencias, el uso del cálculo, etc. Registrar y analizar los resultados experimentales en formas gráficas y matemáticas y sacar conclusiones de las observaciones y los datos obtenidos son fundamentales. El uso del ordenador, tan extendido hoy entre las nuevas generaciones, simplifica estos cálculos (considerados en otros tiempos como tediosos, a base de tablas de logaritmos, ábacos, reglas de cálculo, etc).

5. Convencer al estudiante de que incluso un experimento que aparentemente es poco importante para su futuro profesional, puede contribuir directamente en su desarrollo mental, simplemente por la lógica, análisis o cálculo que lleva consigo.

6. Mejorar la capacidad del alumno en su propia expresión a través de la presentación y exposición de sus resultados, para lo cual el ordenador es una pieza esencial (Aguilar,1983).

7. Las precauciones a observar en los laboratorios de química son de gran importancia, ya que van asociadas al riesgo de materiales peligrosos, pero necesarias para la experiencia y deben los alumnos ser advertidos por su monitor antes de iniciar la manipulación de los mismos. Sólo de este modo adquirirán un concepto real de la relación que existe entre la química de pizarra y la experimental (CBA, 1973).

Si las enseñanzas físico-químicas se realizan con estas líneas de enfoque experimental, los alumnos que sigan con interés los cursos correspondientes, estarán en condiciones:

a) de utilizar más eficazmente sus conocimientos teóricos y hacer con ellos predicciones que podrán ensayar en el laboratorio;

b) considerar si una teoría se aplica a una situación particular y si son posibles ciertas aproximaciones e idealizaciones de la teoría;

c) plantear una o varias experiencias para comprobar el cumplimiento de una determinada teoría;

d) extraer conclusiones de las observaciones y datos obtenidos y evaluar las discrepancias que existen entre la teoría y la experiencia.

Consideramos también que es fundamental para el buen funcionamiento del laboratorio, un equipo de instructores que realicen su trabajo con ilusión y entrega. Un equipo de instructores apáticos y con actitud simplemente mercenaria es el peor enemigo de la enseñanza experimental. Suele resultar fructífero el reclutamiento de instructores posgraduados o de últimos cursos, que son bien recibidos por los alumnos en razón de su edad y status, así como por el hecho de proporcionarles explicaciones a su propio nivel, a los cuales no tienen reparo en discutir o pedir más aclaraciones. 
Desde Galileo y Lavoisier hasta nuestros días, la física y la química experimentales, con los pies puestos en el laboratorio, han sido enseñadas con éxito por los grandes maestros de estas ciencias. Es bien conocido que Hans Christian Oersted hizo su famoso descubrimiento del electromagnetismo en 1820 mientras explicaba una clase a sus alumnos rodeado de pilas, alambres e imanes y afirmaba que todos los avances científicos deben partir de la experimentación. En tiempos más recientes, las clases del profesor Scherrer, en Zurich, eran foco de atracción de todos los físicos amantes de la experimentación. Por último, quiero recordar que Highet en su libro The Art of Teaching (Highet, 1954) al discutir los métodos de los grandes maestros de la historia y, hablando de Jesús, dice: «Como todos los grandes maestros sabía bien el valor de las imágenes (parábolas) y que su pueblo aprendía rápidamente con hechos (milagros)». Era el método experimental llevado al más alto nivel espiritual.

\section{Ciencia fundamental y crecimiento industrial}

Las ciencias fisíco-químicas, con la biología y las matemáticas, constituyen hoy los ingredientes fundamentales de lo que se llama ciencia pura o fundamental. Si analizamos el desarrollo de las naciones industriales en los últimos 200 años, no hay duda de que en la primera mitad del siglo XIX, Inglaterra era la más destacada; los grandes científicos de la investigación fundamental eran ingleses: Maxwell, Faraday, Young, Davy, etc. En la segunda mitad del XIX y principios del XX, Alemania tomó la cabeza y los grandes científicos puros fueron alemanes en su mayoría: Helmholtz, Nernst, Roentgen, Planck, Sommerfeld, Heisenberg, etc. Después de la II Guerra Mundial, los EE.UU predominaron a escala industrial y sus mejores científicos puros fueron Fermi, Oppenheimer, Lawrence, Rabi, McMillan, Feynman, etc. La relación es evidente. El poderío industrial se nutre de grandes físicos, químicos y matemáticos. Estos no surgen por generación espontánea, sino de la excelente formación que recibieron en sus universidades de origen.

Como decía V.F. Weisskopf, «la investigación fundamental fija las normas del pensamiento científico y crea el clima intelectual de la civilización contemporánea. Es el motor que hace circular la sabia de las ideas, no sólo en los laboratorios tecnológicos y las industrias, sino también en todas las actividades culturales» (Weisskopf, 1963)

En el discurso pronunciado en el Vaticano, ante la Academia de las Ciencias, al cumplirse el I Centenario del nacimiento de Albert Einstein (10 de noviembre de 1979), el Papa Juan Pablo II (1980) decía: 


\section{José Aguilar Peris}

«La investigación fundamental debe estar libre de la influencia de los poderes político y económico, los cuales deben cooperar a su desarrollo, pero sin entrometerse en su creatividad, ni someterla a sus propios fines... En cuanto a su segunda vertiente, la ciencia aplicada, debe aliarse con la conciencia del científico, a fin de que el trinomio ciencia-tecnología-conciencia sea la causa del bien verdadero del hombre, al cual va destinado».

No olvidemos que la preparación no adecuada (de bajo nivel) en matemáticas y ciencias fisíco-químicas en aquellos alumnos que desean como fin último la investigación o la integración en una empresa de alta tecnología, dará lugar necesariamente a una frustración que contribuye finalmente a un alto grado de abandono en sus ilusiones. Cada día más, los centros selectos de investigación y las grandes empresas exigen un curriculum de excelencia en sus futuros técnicos y personal directivo, valorando incluso sus conocimientos de economía, ciencias sociales, idiomas, informática, contaminación ambiental, ecología, etc., para mantenerse como líderes en estos nuevos aspectos cada vez más importantes en nuestro entorno.

\section{Las ciencias físico-químicas y la tecnología}

Las ciencias fundamentales fisíco-químicas y la tecnología se apoyan mutuamente en formas muy diversas, a veces cíclicas, como en una simbiosis natural. Un ejemplo de grandes dimensiones, desarrollado en los últimos cien años y que sigue aportándonos ramificaciones sin fin, es el correspondiente al estudio de las partículas elementales y su influencia en el desarrollo de la tecnología más avanzada.

En efecto, el descubrimiento crucial del electrón y los rayos X por J. J. Thomson y W. C. Roentgen no hubiera sido posible sin las bombas de alto vacío y los aparatos espectroscópicos que manejaban los químicos en los últimos años del siglo XIX con fines analíticos. Estos descubrimientos, unidos a la teoría cuántica, puesta en marcha por M. Planck al resolver el problema de la radiación térmica emitida por un cuerpo negro, permitieron que Bohr enunciase su modelo atómico y justificara el origen y la energía de las rayas espectrales. El tubo de rayos catódicos utilizado por Thomson para medir la masa y la carga del electrón constituye esencialmente el tubo y la pantalla de los aparatos de televisión. Podríamos decir, finalmente, que el televisor posee el acelerador de electrones más universal y popular de nuestro mundo.

De estos descubrimientos surgió el concepto de probabilidad de la nube electrónica que rodea el átomo, base esencial que permitió explicar 


\section{Las ciencias físico-químicas y su enseñanza}

la química moderna de los elementos, los láseres y la superconductividad. Con los imanes superconductores, la técnica de la resonancia magnética nuclear se ha convertido en un aparato importante de la medicina actual. Los cables superconductores podrán utilizarse algún día para el transporte de energía eléctrica con un mínimo de pérdida de calor, así como para su almacenamiento mediante bobinas. Las técnicas de detección de partículas, puestas a punto con estos mismos principios, tienen actualmente numerosas aplicaciones en industrias del petróleo, siderurgia, medicina y armamento. Gracias a los detectores de centelleo se apreció casi inmediatamente en toda Europa la explosión del reactor nuclear de Tchernobyl.

Así se abrió la puerta hacia el progreso imparable de la electrónica, la astronomía, la informática y las comunicaciones y también como un efecto de retroalimentación, a profundizar más en el estudio de las partículas elementales. Así se construyeron los grandes colisionadores del CERN, gracias a los cuales se reproducen y se investigan los fenómenos que tuvieron lugar en los primeros segundos después del big-bang, la gran explosión que hace 15000 millones de años puso en marcha el universo. Actualmente los haces de partículas aceleradas se utilizan también en el tratamiento de alimentos, destruyendo todo tipo de bacterias y prolongando así el tiempo de conservación, así como en medicina y en la industria electrónica.

La segunda ola de repercusiones fundamentales del estudio de las partículas elementales tuvo su origen en los trabajos de Becquerel y los esposos Curie, que dieron lugar al descubrimiento de la radiactividad y a nuevos elementos como el radio y el polonio. Hoy los isótopos radiactivos pueden obtenerse artificialmente con múltiples usos en medicina, agricultura e industria. Así por ejemplo, el cáncer de tiroides puede tratarse inyectando al paciente una pequeña cantidad de un isótopo radiactivo del yodo. Este selectivamente se fija en el tiroides y destruye con su radiación las células cancerosas. Igualmente, el camino seguido por un lubrificante en un proceso industrial o el de un fertilizante en un vegetal en crecimiento, pueden seguirse incorporando un isótopo radiactivo, que se localiza mediante un detector. Otra aplicación singular es la datación radiactiva, capaz de determinar la edad de una roca, una momia o unos restos arqueológicos (Courrier du CERN, 1988).

En 1905, Einstein publica su teoría de la relatividad restringida en la que demuestra que la masa puede convertirse en energía e inversamente, fenómeno expresado por la famosa ecuación $E=m c^{2}$, en donde $c$ es la velocidad constante de la luz. Esta relación es la base de la fisión nuclear del uranio-235 y de su aplicación bélica en la II Guerra Mundial. Fi- 
nalizada la guerra, y bajo los auspicios del lema «átomos para la paz», se construyeron los primeros reactores nucleares para la producción de energía eléctrica. Hoy, la esperanza del hombre está en los reactores de fusión, que carecen de residuos radiactivos dañinos. Además, su materia prima, los isótopos del hidrogeno, se encuentra en el mar. Desgraciadamente, su puesta a punto avanza lentamente y los más optimistas piensan que la energía de fusión no estará a disposición del hombre hasta la segunda mitad del siglo actual.

\section{El método histórico en la enseñanza de las ciencias físico- químicas}

En la mayor parte de los textos introductorios de física y química, es corriente encontrar frases como éstas: «la primera definición de elemento químico fue dada por Boyle en 1667»; «Roemer determinó por vez primera la velocidad de la luz en 1676»; «Lavoisier expuso el principio de conservación de la masa en 1770»; «en 1899 las primeras emisiones radioeléctricas (T.S.H.) ideadas por Marconi atravesaron el Canal de la Mancha»; «en 1905 Einstein publicó su teoría de la relatividad restringida», etc.

Estos datos son necesarios, pero si no se amplían con algunas referencias más concretas sobre los hechos esenciales que rodearon el fenómeno en cuestión y las características humanas del científico citado, es decir, una pequeña historia del mismo, todo quedará pronto olvidado. El alumno, si es aplicado, superará los exámenes correspondientes, pero nunca llegará a comprender la importancia de la ciencia en el desarrollo histórico de la civilización occidental.

Cierto es que hoy existen textos de «Ciencia para no científicos», en donde el método histórico es el núcleo fundamental de la exposición, pero naturalmente carecen del desarrollo científico y técnico que se exige a los alumnos que siguen los cursos de enseñanza media o universitaria. Incluir ambos contenidos bien desarrollados, ciencia e historia, en un solo texto, tampoco parece prudente. Sin embargo, existen también libros de texto de materias físico-químicas (como el citado PSSC, el proyecto Harvard, los textos de Tipler, etc) que poseen el nivel adecuado para la formación científica rigurosa, pero que introducen en forma de ensayos o preludios, lecturas útiles sobre biografías y descubrimientos, polémicas de carácter histórico, etc, que alivian esta falta de información y humanismo a los que nos estamos refiriendo.

El mismo Pasteur, hace ya muchos años, decía (French, 1989):

«Existen dos métodos diferentes para describir un descubrimiento científico. Uno consiste en presentar la ley de comportamiento y de- 


\section{Las ciencias físico-químicas y su enseñanza}

mostrarla en su expresión actual sin detenerse en la forma en que se desarrolló. El otro es aquél que trata de transportar mentalmente a sus alumnos al periodo en que el descubrimiento se realizó, los primeros esfuerzos para la comprensión del fenómeno y su evolución experimental y teórica hasta el instante en que comprueba la aplicación universal de su propia teoría».

Este método histórico es el que se expone en el texto de G. Holton (1988), sin olvidar un elevado rigor en el desarrollo de los conceptos científicos.

\section{Educación y excelencia}

La máxima responsabilidad de un profesor es transmitir con honestidad y entusiasmo sus conocimientos y experiencia a sus alumnos. Esta instrucción por medio de la acción docente, que dirige y desarrolla las facultades intelectuales del alumno mediante lecciones magistrales, ejercicios, proyectos, y una buena dosis de humanidad, es la base del éxito de la educación, lo mismo en ciencias jurídicas o económicas que en ciencias fisíco-químicas.

Los educadores no solo tienen la importante misión de informar, sino también la de crear en su clases el clima necesario para que el estudio se convierta en una mezcla de curiosidad, deseo de aprender y satisfacción personal. Un joven universitario suele sentirse más atraído por los grandes enigmas de las ciencias de la naturaleza que por los problemas prácticos. Esta curiosidad, cultivada por su profesor y que es típica en los alumnos mejor dotados, si no se malogra por causas externas, fructificará en excelentes graduados.

Para terminar quiero recordar las palabras que Einstein dirigió a un grupo de jóvenes universitarios que fueron a visitarle en su residencia de Princeton (N.J., EE.UU), poco antes de su muerte:

«Nunca toméis el estudio como una obligación, sino como una maravillosa oportunidad de aprender. Si aprovecháis esta oportunidad, experimentareis una gran alegría personal, no comparable a ninguna otra de tipo material. Además, con ello beneficiareis a la sociedad, a la que pertenece vuestro trabajo futuro».

\section{Bibliografía}

AGUILAR, J. (1965): «La enseñanza de la física y su problemática». Arbor, 234, pp 5-33. 
AGUilar, J. (1983): «La enseñanza de la física experimental». Phina (publicaciones Phiwe), 183, pp 4-6.

Bouyssy, A., Davier, M. y GatTy, B. (1987): Phisique pour les sciences de la vie. París: Ed. Belin.

CBA (1973): Chemical Bond approach project. Vols I y II. Barcelona: Editorial Reverté. Chapman, S. (1959): How to study Physics. Boston (Mass.): Addison-Wesley Pub. Co. COURRIER DU CERN (1988): «Ce que la Physique des particles apporte a la technologie». Novembre 1988, pp 1-18.

FRENCH, A.P. (1989): «Learning from the past, looking to the future». American Journal of Physics, 56(6)110.

Glasser, O. (1944): Medical Physics. Chicago: Yearbook Pub. Inc.

HigheT, G. (1954): The art of teaching. New York: Ed. Knopf.

Holton, G. (1988): Introducción a los conceptos y las teorías de las Ciencias Físicas. Barcelona: Ed. Reverté.

JUAN PABLO II (1980): «Alocución de Su Santidad». Courrier du CERN, abril, p. 65.

WEISSKOPF, V.F. (1963): «Quel est l'interêt de la science pure?». Courrier du CERN, marzo, p. 99. 